\title{
Kondo Resonance in a Mesoscopic Ring Coupled to a Quantum Dot: Exact Results for the Aharonov-Bohm-Casher Effects
}

\author{
H.-P. Eckle, ${ }^{1,2}$ H. Johannesson, ${ }^{3}$ and C. A. Stafford ${ }^{4}$ \\ ${ }^{1}$ School of Physics, The University of New South Wales, Sydney, 2052, Australia \\ ${ }^{2}$ Department of Physics, University of Jyväskylä, FIN-40351 Jyväskylä, Finland \\ ${ }^{3}$ Institute of Theoretical Physics, Chalmers University of Technology and Göteborg University, SE 412 96 Göteborg, Sweden \\ ${ }^{4}$ Department of Physics, University of Arizona, 1118 E. 4th Street, Tucson, AZ 85721
}

\begin{abstract}
We study the persistent currents induced by both the Aharonov-Bohm and Aharonov-Casher effects in a one-dimensional mesoscopic ring coupled to a side-branch quantum dot at Kondo resonance. For privileged values of the Aharonov-Bohm-Casher fluxes, the problem can be mapped onto an integrable model, exactly solvable by a Bethe ansatz. In the case of a pure magnetic AharonovBohm flux, we find that the presence of the quantum dot has no effect on the persistent current. In contrast, the Kondo resonance interferes with the spin-dependent Aharonov-Casher effect to induce a current which, in the strong-coupling limit, is independent of the number of electrons in the ring.
\end{abstract}

PACS numbers: 72.15.Qm, 73.23.Hk, 85.35.Be

The Kondo effect - where the interaction between a local spin and free electrons produces a strongly-correlated state below a characteristic temperature $T_{K}$ - has become one of the paradigms in the study of correlated electron behavior [1]. In a recent experimental breakthrough [2], a tunable Kondo effect was observed in ultra small semiconductor quantum dots connected capacitively to a gate and via tunnel junctions to electrodes. By sweeping the gate voltage, the dot's highest spin-degenerate level $\epsilon_{d}$ can be tuned relative to the chemical potential $\mu$ of the leads. This level is occupied by a single electron when $\epsilon_{d} \leq \mu-\Gamma_{d}$, with $\Gamma_{d}=2 \pi \sum_{k}\left|V_{k}\right|^{2} \delta\left(\epsilon_{d}-\epsilon_{k}\right)$ the oneparticle resonance width of the dot, and $V_{k}$ the tunneling matrix elements through the junction barriers. Below a temperature $T_{K} \sim \exp \left(-\pi\left|\mu-\epsilon_{d}\right| / \Gamma_{d}\right)$ the resulting free spin on the dot forms a singlet with the electron spins in the leads via virtual co-tunneling processes. A fingerprint of this strongly correlated state is the dramatic enhancement of the local spectral density at the Fermi level. As predicted theoretically [3, 边 and as seen in the experiments [2], this makes the dot transparent to electron transport when $T \ll T_{K}$.

An interesting problem is how the persistent current (PC) of a multiply connected system coupled to a quantum dot is affected by a Kondo resonance. A PC is the equilibrium response [5] to a magnetic AharonovBohm (AB) flux [8] and/or an Aharonov-Casher "flux" [9] of a charged wire piercing the system. In contrast to ordinary (nonequilibrium) currents, as measured in the experiments referred to above, a PC requires for its existence that an electron maintains its phase coherence while circling the ring, and should thus be sensitive to scattering in the quantum dot 10. The $\mathrm{PC}$ of a onedimensional (1D) ring coupled to a quantum dot was previously investigated by Büttiker and one of the authors [11], considering two different topologies: one in which the electron had to tunnel through the quantum dot in order to encircle the flux (embedded dot), and one in which an intact ring was coupled via a tunnel barrier to an adjacent quantum dot (sidebranch dot). However, in Ref. [11], the energy level spacing $\delta$ in the mesoscopic ring was taken to be much greater than $T_{K}$, so that although a singlet state was formed between the electron on the dot and those within the ring, this state had more in common with a (two-body) chemical bond than with a true (many-body) Kondo resonance: the finite-size effects implied by $\delta \gg T_{K}$ here suppress the Kondo effect.

Recently, the problem of a ring with an embedded quantum dot has been revisited by a number of authors 12 14, and the Kondo scaling with $\delta$ has been investigated [13]. Based on a cluster calculation in the grand canonical ensemble, Ferrari et al. 12. claim that the PC is strongly enhanced by the Kondo effect, scaling as $L^{-1 / 2}$ when $\delta>T_{K}$ (this includes the regime studied in Ref. [11], where no appreciable enhancement was found). In contrast, using a variational approach, Kang and Shin 113 find that the Kondo-assisted PC is comparable to that of a perfect ring for an even number of electrons, and strongly suppressed for an odd number of electrons. Other related results have also been obtained [14, 15. Clearly, the situation calls for a more definitive analysis to clarify the underlying physics of this class of problems.

In this Letter, we investigate the PC in a $1 \mathrm{D}$ mesoscopic ring coupled via tunneling to a sidebranch quantum dot in the Kondo regime, with $\delta \leq T_{K}$ (opposite to the limit $\delta \gg T_{K}$ considered in [1]). We exploit the fact that the PC to leading order in 1/L effectively involves only processes close to the Fermi level [16,17]: In a 1D ring, the system responds to the $\mathrm{AB}$ and/or $\mathrm{AC}$ fluxes by virtually creating and annihilating states at the left and right Fermi points, and this leads to a change in the energy on a mesoscopic scale, producing a PC. Since the coupling to the dot via the Kondo exchange influences only states at the Fermi level, this allows us to construct 
an effective model valid in the neighborhood of the Fermi points. The model turns out to be exactly solvable for a discrete set of values of the $\mathrm{AB}$ and/or $\mathrm{AC}$ fluxes, for which we can calculate the PC exactly. Via adiabatic continuation, we conclude that the $\mathrm{AB} \mathrm{PC}$ is insensitive to the presence of the quantum dot for any weak AB flux, thus extending the result found in 11 into the Kondo regime. In contrast, with an $\mathrm{AC}$ flux we find that the Kondo resonance produces an imbalance between rightand left moving charge carriers, leading to a net AC PC.

A quantum dot connected to a 1D electron reservoir (in our case, a mesoscopic ring) is conventionally described by the Anderson impurity model [3, 4]:

$H=\sum_{\alpha} \epsilon_{d} d_{\alpha}^{\dagger} d_{\alpha}+U n_{\uparrow} n_{\downarrow}+\sum_{k, \alpha} \epsilon_{k} c_{k \alpha}^{\dagger} c_{k \alpha}+\left(V_{k} c_{k \alpha}^{\dagger} d_{\alpha}+\right.$ H.c. $)$

where $d_{\alpha}^{\dagger}$ creates an electron on the dot with spin $\alpha, c_{k \alpha}^{\dagger}$ creates an electron of energy $\epsilon_{k}$ in the ring, and $n_{\alpha}=$ $d_{\alpha}^{\dagger} d_{\alpha}$. Here we consider only a single spin-degenerate level $\epsilon_{d}$ within the quantum dot. We shall take the charging energy $U \rightarrow \infty$, as is appropriate for quantum dots where $U \sim 1 \mathrm{meV}$ and $\Gamma_{d} \sim 1-10 \mu \mathrm{eV} 18$.

When $\epsilon_{d} \ll \mu-\Gamma_{d}$, the dot is singly occupied, i.e., $\sum_{\alpha}\left\langle d_{\alpha}^{\dagger} d_{\alpha}\right\rangle=1$, and the Anderson model can be mapped onto a Kondo Hamiltonian via a Schrieffer-Wolff transformation [1]. To have a faithful representation we require that $\delta \leq T_{K}$, which ensures a finite DOS at the Fermi level and hence a fully developed Kondo resonance [19]. Passing to a real-space continuum description, we obtain

$$
\begin{aligned}
H= & -\frac{\hbar^{2}}{2 m_{e}} \sum_{\alpha} \int_{0}^{L} d x \psi_{\alpha}^{\dagger}(x) \partial_{x}^{2} \psi_{\alpha}(x) \\
& +\lambda \sum_{\alpha, \beta} \psi_{\alpha}^{\dagger}(0) \boldsymbol{\sigma}_{\alpha \beta} \psi_{\beta}(0) \cdot \boldsymbol{S}
\end{aligned}
$$

where the antiferromagnetic spin exchange takes the value $\lambda \sim\left|V_{k_{F}}\right|^{2} /\left|\epsilon_{d}\right|$ (with the chemical potential $\mu$ taken as the zero of energy), $L$ is the circumference of the ring, $\boldsymbol{S}$ is the spin-1/2 operator of the electron in the dot (located at $x=0$ ), and $\psi_{\alpha}(x)$ is an electron field with spin index $\alpha= \pm 1$. Next, we thread the ring with a magnetic flux $\Phi$, producing an Aharonov-Bohm effect [8]. In addition, to probe the spin-dependent equilibrium response of the system, we pierce the ring with a charged wire of charge $\tau$ per unit length. This will cause an Aharonov-Casher effect [9] driven by the relativistic spin-orbit interaction due to the electric field from the wire. The quantum phases from the combined Aharonov-Bohm-Casher (ABC) effects can be encoded, via a gauge transformation [5], in the twisted boundary conditions $\psi_{\alpha}(L)=e^{i \phi_{\alpha}} \psi_{\alpha}(0)$, where

$$
\phi_{\alpha}=2 \pi\left(\frac{\Phi}{\Phi_{0}}+\alpha \frac{\tau}{\tau_{0}}\right), \quad \alpha= \pm 1 .
$$

Here $\Phi_{0}=h c / e$ is the elementary magnetic flux quantum and $\tau_{0}=h c / \mu_{e}$ its electromagnetic dual, $\mu_{e}$ being the electron magnetic moment.

Since the essential physics is confined to a small region around the left and right Fermi points, we can linearize the spectrum around $\pm k_{\mathrm{F}}$ 17, 19, and introduce left $(l)$ and right $(r)$ moving chiral fields:

$$
\psi_{\alpha}(x) \sim \mathrm{e}^{-i k_{\mathrm{F}} x} \psi_{l, \alpha}(x)+\mathrm{e}^{i k_{\mathrm{F}} x} \psi_{r, \alpha}(x) .
$$

Furthermore we introduce a basis of definite parity fields (Weyl basis):

$$
\psi_{\mathrm{e} / \mathrm{o}, \alpha}(x)=\frac{1}{\sqrt{2}}\left(\psi_{r, \alpha}( \pm x) \pm \psi_{l, \alpha}(\mp x)\right),
$$

with $\psi_{\mathrm{e}}$ an even-parity, right-moving electron field, and $\psi_{\mathrm{o}}$ an odd-parity, left-moving field. In the Weyl basis, the linearized Hamiltonian takes the form $H=H_{0}^{\text {odd }}+$ $H_{0}^{\text {even }}+H_{\text {imp }}^{\text {even }}$, where

$$
H_{0}^{\mathrm{even} / \mathrm{odd}}=\mp \frac{v_{\mathrm{F}}}{2 \pi} \sum_{\alpha} \int_{0}^{L} d x \psi_{\mathrm{e} / \mathrm{o}, \alpha}^{\dagger}(x) i \partial_{x} \psi_{\mathrm{e} / \mathrm{o}, \alpha}(x)
$$

describe free Dirac electrons with velocity $v_{\mathrm{F}}$, and the impurity contribution is diagonal in the channel index:

$$
H_{\mathrm{imp}}^{\mathrm{even}}=\lambda \sum_{\alpha, \beta} \psi_{\mathrm{e}, \alpha}^{\dagger}(0) \boldsymbol{\sigma}_{\alpha \beta} \psi_{\mathrm{e}, \beta}(0) \cdot \boldsymbol{S} .
$$

We recognize $H_{K}^{\text {even }} \equiv H_{0}^{\text {even }}+H_{\text {imp }}^{\text {even }}$ as the chiral Hamiltonian of the spin-1/2 Kondo model [1].

While the even and odd parity channels are decoupled in the Hamiltonian, they become connected again by the twisted boundary conditions:

$$
\left(\begin{array}{c}
\psi_{\mathrm{e}, \alpha}(L) \\
\psi_{\mathrm{o}, \alpha}(L)
\end{array}\right)=\left(\begin{array}{cc}
\cos \phi_{\alpha} & i \sin \phi_{\alpha} \\
-i \sin \phi_{\alpha} & \cos \phi_{\alpha}
\end{array}\right)\left(\begin{array}{c}
\psi_{\mathrm{e}, \alpha}(0) \\
\psi_{\mathrm{o}, \alpha}(0)
\end{array}\right),
$$

where in (3) we have taken $k_{\mathrm{F}}=(2 \pi / L) n$, with $n$ an integer. At the special values $\phi_{\alpha}=f_{\alpha} \pi$, with $f_{\alpha}$ an integer, however, this matrix reduces to a multiple of the unit matrix, and the even and odd parity states decouple from each other entirely. For the corresponding values of the ABC fluxes, the model becomes integrable and $H_{K}^{\text {even }}$ can be solved by a Bethe ansatz 20]. Thus, our original problem has been mapped onto an exactly solvable problem consisting of a left-moving odd-parity branch of free Dirac electrons, together with a (decoupled) rightmoving even-parity branch, described by an integrable 1D Kondo model.

Having constructed an effective model valid near the Fermi level, we need to properly define the (charge) PC of the system using only Fermi level properties. This can be done as follows: We introduce the excess numbers $\Delta N_{r / l}$ of particles (holes) on the right/left dispersion branches due to the Aharonov-Bohm-Casher fluxes: 


$$
\Delta N_{r / l}\left(\phi_{\alpha}\right)=\frac{L}{2 \pi}\left[\left|k_{r / l, \mathrm{~F}}\left(\phi_{\alpha}\right)\right|-\min \left(\left|k_{l, \mathrm{~F}}(0)\right|, k_{r, \mathrm{~F}}(0)\right)\right],
$$

with $k_{r / l, F}$ flux-dependent momenta associated with the highest occupied level on the respective branch. (For free electrons, $\left.k_{r / l, \mathrm{~F}}=\left[ \pm 2 \pi n_{r / l, \max }+\phi_{\alpha}\right] / L\right)$. This correctly accounts for the average number of surplus currentcarrying particles $\left(\Delta N_{r / l}>0\right)$ and holes $\left(\Delta N_{r / l}<0\right)$ due to the fluxes when the total particle number $N$ is fixed and hence the chemical potential is free to vary between the highest occupied and lowest unoccupied levels. Since the charge velocity $v_{\mathrm{F}}$ is unrenormalized by interactions in this model [20], each current-carrying state contributes with $\pm e v_{\mathrm{F}} / L$ to $I(\Phi, \tau)$ (with the sign determined by the branch $r / l$ and the sign of $\phi_{\alpha}$ ), and we thus obtain

$$
I(\Phi, \tau)=-\frac{e v_{\mathrm{F}}}{L} \sum_{\alpha= \pm 1}\left(\Delta N_{r}\left(\phi_{\alpha}\right)-\Delta N_{l}\left(\phi_{\alpha}\right)\right) .
$$

When the total number $N$ of electrons in the ring is even (which, for simplicity, is the case we shall focus on below), we still have to distinguish two cases: When $N=4 n+2$, with $n$ an integer, $k_{r, F}(0)=\left|k_{l, F}(0)\right|$, while for $N=4 n$ the lifting of the groundstate degeneracy due to the flux in this case implies that $k_{r, F}\left(0_{ \pm}\right)=\left|k_{l, F}\left(0_{ \pm}\right)\right| \mp 2 \pi / L$ in the limit $\phi_{\alpha} \rightarrow 0_{ \pm}$. This leads to the well-known parity effect [5]:17,7] (here for an even number of spinful electrons), as is easily verified from (7).

Given (7), the problem is now reduced to calculating how the excess numbers depend on the ABC fluxes in the presence of a Kondo dot. To do this, we apply the techniques of the Bethe ansatz for finite systems, developed previously for the 1D Hubbard model [21]. As we have already noted, our model is integrable for $\phi_{\alpha}=f_{\alpha} \pi$, with $f_{\alpha}$ an integer. The nested Bethe ansatz equations (BAE) which diagonalize $H$ in this case are

$$
\begin{gathered}
L k_{n_{l}}=-2 \pi n_{l}+f_{c} \pi+\left(\frac{2 M_{\mathrm{o}}}{N_{\mathrm{o}}}-1\right) f_{s} \pi+\frac{2 \pi}{N_{\mathrm{o}}} \sum_{\delta=1}^{M_{\mathrm{o}}} J_{\delta}, \\
L k_{n_{r}}=2 \pi n_{r}+f_{\downarrow} \pi+\sum_{\gamma=1}^{M_{\mathrm{e}}}\left[\theta\left(2 \Lambda_{\gamma}-2\right)-\pi\right],
\end{gathered}
$$$$
N_{\mathrm{e}} \theta\left(2 \Lambda_{\gamma}-2\right)+\theta\left(2 \Lambda_{\gamma}\right)=2 \pi I_{\gamma}+\left(f_{\uparrow}-f_{\downarrow}\right) \pi+\sum_{\delta=1}^{M_{\mathrm{e}}} \theta\left(\Lambda_{\gamma}-\Lambda_{\delta}\right),
$$

where $k_{n_{l}}$ are the pseudomomenta characterizing the $N_{\mathrm{o}}$ odd-parity left movers which decouple from the impurity, $M_{\mathrm{o}}$ of which have spin down, and $k_{n_{r}}$ are pseudomomenta characterizing the $N_{\mathrm{e}}$ even-parity right movers, with $M_{\mathrm{e}}$ counting the number of down spins in this sector. The quantum numbers $n_{l}, n_{r}, I_{\gamma}$ and $J_{\delta}$ specifying the state take integer or half-odd integer values depending on the values of $M_{\mathrm{e} / \mathrm{o}}$ and $N_{\mathrm{e} / \mathrm{o}}$, while $\left\{\Lambda_{\gamma}, \gamma=1, \ldots, M_{\mathrm{e}}\right\}$ are a set of auxiliary variables known as spin-rapidities. The scattering phase shifts are given by $\theta(x)=2 \tan ^{-1}(x / c)$, with $c=2 \lambda /\left(1-3 \lambda^{2} / 4\right)$ playing the role of an effective coupling constant. We have also defined $f_{c, s}=\left(f_{\uparrow} \pm\right.$ $\left.f_{\downarrow}\right) / 2$. The first BAE simply gives the quantum numbers of free, chiral electrons, written in the Bethe ansatz basis. The second BAE describes the charge degrees of freedom in the even channel (holons), while the third describes the spin degrees of freedom in the even channel (spinons).

Consider first the case where only a magnetic flux threads the ring, with a spin-independent integrable $\mathrm{AB}$ phase $f_{\uparrow}=f_{\downarrow}=f$. To be explicit, we choose $N=4 n+2$ with $M_{\mathrm{e} / \mathrm{o}}=\left(N_{\mathrm{e} / \mathrm{o}} \pm 1\right) / 2$, where $N_{\mathrm{e}}=N_{\mathrm{o}}$. For this case, the groundstate is characterized by integer-spaced quantum numbers $\left\{n_{l}, J_{\delta}, n_{r}, I_{\gamma}\right\}$ in the symmetric ranges $-\left(N_{\mathrm{o}}-1\right) / 2 \leq n_{l} \leq\left(N_{\mathrm{o}}-1\right) / 2,-\left(M_{\mathrm{o}}-1\right) / 2 \leq$ $J_{\delta} \leq\left(M_{\mathrm{o}}-1\right) / 2,-\left(N_{\mathrm{e}}-1\right) / 2 \leq n_{r} \leq\left(N_{\mathrm{e}}-1\right) / 2$, and $-\left(M_{\mathrm{e}}-1\right) / 2 \leq I_{\gamma} \leq\left(M_{\mathrm{e}}-1\right) / 2$. Once a set of spin rapidities $\Lambda_{\gamma}$ is given, the momenta $k_{n_{r}}$ are determined, and the excess numbers on the right and left branches can be derived. With $f_{c}=f$ and $f_{s}=0$, it becomes immediately clear from the BAE that the total scattering phase shift from the dot is independent of $f$, and we thus obtain that $\Delta N_{r}(f)=-\Delta N_{l}(f)=f / 2$. For an even number of electrons, the sum in (7) can be written as $2\left(\Delta N_{r}\left(f_{c}, f_{s}\right)-\Delta N_{l}\left(f_{c}, f_{s}\right)\right)$, and we obtain $I(\Phi, 0)=-\left(4 e v_{\mathrm{F}} / L\right)\left(\Phi / \Phi_{0}\right)$ at the integrable points $\Phi / \Phi_{0}=f / 2$. This is the same PC as that for an ideal ring of free electrons. The analogous analysis for $N=4 n, N=4 n+1$ and $N=4 n+3$ reveals that for all cases the $A B P C$ is unaffected by the Kondo resonance at the integrable points $\Phi / \Phi_{0}=f / 2$.

It should be pointed out that the PC we have calculated is the current carried by the state which evolves adiabatically from the ground state at $\Phi=0$ as $\Phi$ is turned on. Generically, there may be one or more level crossings 22 between $f=0$ and $f=1$, so that this state may not be the absolute ground state at e.g. $f=1$. Notwithstanding this proviso, it is clear from the following argument that the above result for $I(\Phi, 0)$ must hold for small values of $\Phi / \Phi_{0}$ : By symmetry, the PC is an odd function of $\Phi$ and is analytic, except at values of $\Phi$ corresponding to level crossings. Considering again the case $N=4 n+2$, there is no level crossing at $\Phi=0$. The leading mesoscopic behavior of the PC is then $I(\Phi, 0)=-D_{c} \Phi / L+\mathcal{O}\left(\Phi^{3} / L^{3}\right)$, where $D_{c}$ is the charge stiffness. This holds on general grounds, independent of whether the model is integrable or not. To determine the charge stiffness, however, we only need to consider the state which evolves adiabatically 23] from the ground state at $f=0$, specified above, as $\Phi$ is increased. Thus the above result for $I(\Phi, 0)$ is seen to hold for small values of $\Phi / \Phi_{0}$ as well.

Our result for the $\mathrm{AB}$ PC suggests that spin-charge separation holds generically at the mesoscopic scale of 
this system. The phase shift acquired by scattering off the dot in the even parity channel affects only the spin content of an incoming electron 20], but not the charge which contributes to the PC. This may explain why our result for a ring is different from that obtained for a sidebranch dot connected to an open quantum wire [24: there it was found that the $\pi / 2$ phase shift due to the Kondo resonance suppresses the transmission completely. However, in an open system where single electrons can be added and subtracted (via a source and drain), the holons and spinons - which are the relevant low-energy degrees of freedom in the correlated BA basis - have to be recombined in such a way as to mimic single-electron excitations, and this makes the transmission susceptible to spinon scattering 25,26,. No such constraint is expected to apply to a correlated closed system, and the AB PC - carried by the holons - can flow unperturbed by scattering events in the spin sector.

What then happens to the PC due to a spin-dependent AC flux? Although dynamical spin-charge separation still holds at the integrable points - as is evident from the one-dimensional Kondo Hamiltonian in (6) - the spindependent phase induced by the AC flux may now carry information from the spin sector into the charge sector, hence affecting the PC. The analysis of the BAE in the case of general spin-dependent fluxes (however, still satisfying $\phi_{\alpha}=f_{\alpha} \pi$ with $f_{\alpha}$ an integer) is more involved. However, in the strong-coupling limit $c \rightarrow \infty$ and with a pure $\mathrm{AC}$ flux (i.e. with $f_{\uparrow}=-f_{\downarrow}=f$ ) we can still obtain an analytic solution. For this case, $\theta\left(2 \Lambda_{\gamma}-2\right) \rightarrow \theta\left(2 \Lambda_{\gamma}\right)$ in the BAE, and one may algebraically solve for the excess numbers. Again specializing to the case $2 N_{\mathrm{e}}=2 N_{\mathrm{o}}=N=4 n+2$ with $M_{\mathrm{e} / \mathrm{o}}=\left(N_{\mathrm{e} / \mathrm{o}} \pm 1\right) / 2$, we obtain

$$
I(0, \tau)=2 \frac{e v_{\mathrm{F}}}{L} \cdot \frac{\tau}{\tau_{0}}\left(\frac{1}{N_{\mathrm{o}}}\right), \quad c \rightarrow \infty .
$$

Since $v_{F} \sim N_{\mathrm{o}}$, the current $I(0, \tau)$ is independent of the number of electrons in the ring, and only depends on the charge density $\tau$ and the ring's circumference $L$. Eq. (8) is to be compared to the case of an ideal ring with an equal number of up- and down-spin electrons, for which case there is no AC PC 27]. This is easily verified in the present formalism by simply removing the quantum dot scattering term $\theta\left(2 \Lambda_{\gamma}\right)$ from the BAE. Solving for the PC one obtains $I(0, \tau)=-\left(2 e v_{\mathrm{F}} / L\right)\left(\tau / \tau_{0}\right)\left(N_{\mathrm{e}}^{-1}-N_{\mathrm{o}}^{-1}\right)=0$.

The result in (8) strikingly exhibits the nondynamical nature of the AC effect: In the strong-coupling limit the dot effectively screens the spin content of an electron, and via the $\mathrm{AC}$ boundary condition this causes the charge of an electron in the even parity, right-moving sector to get trapped. It is crucial to realize that this happens in the absence of any interaction term in the Hamiltonian containing the charge density. As a result, the numbers of current carrying states on the right and left branches become unbalanced, leading to a net AC persistent current.
In conclusion, we have shown how a one-dimensional mesoscopic ring of free electrons coupled by a tunnel junction to a quantum dot at a Kondo resonance can be described by an exactly solvable model for certain privileged values of the Aharonov-Bohm-Casher fluxes piercing the ring. This allows for an exact nonperturbative analysis of the relevant physics, in particular how the $\mathrm{PC}$ is influenced by a Kondo resonance.

We thank N. Andrei, R. Egger, H. Grabert and A. Zvyagin for discussions. This work was supported in part by the Finnish Academy and the Deutscher Akademischer Austauschdienst. HPE acknowledges support from the Australian Research Council, HJ from the Swedish Natural Science Research Council, and CAS from NSF grant DMR0072703.

[1] See e.g. A. C. Hewson, The Kondo Problem to Heavy Fermions (Cambridge University Press, Cambridge, 1993).

[2] D. Goldhaber-Gordon et al., Nature 391, 156 (1998); S. M. Cronenwett et al., Science 281, 540 (1998); F. Simmel et al., Phys. Rev. Lett. 83, 804 (1999); W. G. van der Wiel et al., Science 289, 2105 (2000).

[3] L. I. Glazman and M. E. Raikh, JETP Lett. 47, 452 (1988); T. K. Ng and P. A. Lee, Phys. Rev. Lett. 61, 1768 (1988).

[4] Y. Meir, N. S. Wingreen, and P. A. Lee, Phys. Rev. Lett. 70, 2601 (1993); N. S. Wingreen and Y. Meir, Phys. Rev. B 49, 11040 (1994).

[5] N. Byers and C. N. Yang, Phys. Rev. Lett. 7, 46 (1961).

[6] M. Büttiker et al., Phys. Lett. A96, 365 (1983).

[7] For a review, see e.g. A. A. Zvyagin and I. V. Krive, Low Temp. Phys. 21, 533 (1995).

[8] Y. Aharonov and D. Bohm, Phys. Rev. 115, 485 (1959).

[9] Y. Aharonov and A. Casher, Phys. Rev. Lett. 53, 319 (1984).

[10] A. Yacoby, et al., Phys. Rev. Lett. 74, 4047 (1995).

[11] M. Büttiker and C. A. Stafford, Phys. Rev. Lett. 76, 495 (1996).

[12] V. Ferrari et al., Phys. Rev. Lett. 82, 5088 (1999).

[13] K. Kang and S.-C. Shin, Phys. Rev. Lett. 85, 5619 (2000).

[14] P. S. Deo and A. M. Jayannavar, cond-mat/0006035.

[15] A. A. Zvyagin and T. V. Bandos, Low Temp. Phys. 20, 222 (1994).

[16] H.-F. Cheung et al., Phys. Rev. B 37, 6050 (1988).

[17] D. Loss, Phys. Rev. Lett. 69, 343 (1992); A. O. Gogolin and N. V. Prokof'ev, Phys. Rev. B 50, 4921 (1994).

[18] When $U$ decreases below $W$, the band width of the electrons in the ring, it just trades place with $W$ in the scaling expresion for the Kondo temperature, but otherwise does not affect the relevant physics (see Ref. [4).

[19] W. B. Thimm, J. Kroha, and J. von Delft, Phys. Rev. Lett. 82, 2143 (1999).

[20] For a recent review, see e.g. N. Andrei in Series on Modern Condensed Matter Physics - Vol. 6, 458 (World Scientific, Singapore, 1992), Eds. S. Lundquist, et al.

[21] F. Woynarovich and H.-P. Eckle, J. Phys. A: Math. Gen. 
20, L443 (1987); H.-P. Eckle, in Strongly interacting fermions and high $-T_{c}$ superconductivity, B. Douçot and J. Zinn-Justin, eds., Proc. Les Houches 1991, Session LVI, (North-Holland, Amsterdam, 1995).

[22] N. Yu and M. Fowler, Phys. Rev. B 45, 11795 (1992); C. A. Stafford and A. J. Millis, Phys. Rev. B 48, 1409 (1993).

[23] F. D. M. Haldane, Phys. Lett. 81A, 153 (1981).

[24] K. Kang, et al., cond-mat/0009235.

[25] P. Fröjdh and H. Johannesson, Phys. Rev. Lett. 75, 300 (1995); Phys. Rev. B 53, 3211 (1996).

[26] C. A. Stafford, Phys. Rev. B 48, 8430 (1993).

[27] A. V. Balatsky and B. L. Altshuler, Phys. Rev. Lett. 70, 1678 (1993). 